\title{
Estructura y Composición Florística del Bosque Seco Tropical en los Montes de María (Sucre - Colombia)
}

\section{Structure and floristic composition of tropical dry forest in the Montes de María (Sucre - Colombia)}

\author{
Fran Herazo Vitola a \\ Jorge Mercado Gómez ${ }^{\text {b* }}$ \\ $\&$ Humberto Mendoza Cifuentes ${ }^{c}$
}

Recepción: 9 de noviembre de 2016

Aceptación: 30 de diciembre de 2016

\begin{abstract}
The structure and arboreal composition was characterized in fragments of tropical dry forest located in the Montes de María in the state of Sucre (Colombia). Seven localities were sampled through the RAP method and proposed modifications. A total of 363 species were recorded in 196 genera and 65 families. The families with the greatest ecological importance were Capparaceae, Fabaceae and Rubiaceae.The species with higher IVI were Simira cordifolia, Trichilia acuminate and Myrcia fallax. With respect to the floristic structure, the growth habit shrubby was the most predominant, followed by trees and herbs as the least representative. The altimetric, diametrical classes and basal areas indicated that the localities of "Paraíso", "Pajarito", "Garrapata" and "Casa Amarilla uno" were the better state, since they accumulated the highest number in the class II (adult trees between 5,1 and $10 \mathrm{~m}$.) and larger basal area, class III (timber trees between 22 and $46 \mathrm{~cm}$ ).
\end{abstract}

Keywords: Fabaceae, richness, composition, Colombia.

\section{Resumen}

Se caracterizó la estructura y composición en fragmentos de bosque seco tropical ubicado en los Montes de María en el departamento de Sucre (Colombia). Siete localidades fueron muestreadas a través del método RAP y modificaciones propuestas. Un total de 363 especies fueron registradas en 196 géneros y 65 familias. Las familias con mayor importancia ecológica fueron Capparaceae, Fabaceae y Rubiaceae. Las especies con mayor IVI fueron Simira cordifolia, Trichilia acuminata y Myrcia fallax. Con respecto al hábito de crecimiento, el arbustivo fue el más predominante, seguido por árboles y el menos representativo fue el de las hierbas. Las clases altimétricas, diamétricas y área basal señalaron que las localidades de Paraíso, Pajarito, Garrapata y Casa Amarilla uno fueron las que presentaron mejor estado, ya que estas acumularon el mayor número de ínvidos en la clase II (árboles adultos entre 5,1 y $10 \mathrm{~m}$.) y con mayor área basal, clase III (arboles maderables entre 22 y $46 \mathrm{~cm}$ ).

Palabras clave: Fabaceae, riqueza, composición, Colombia.

a Maestría en Biología. Grupo Evolución y Sistemática Tropical. Universidad de Sucre, Carrera 28 No. 5-267. Sincelejo, Colombia. fran.herazo@unisucre.edu.co.

b Departamento de Biología y Química. Grupo Evolución y Sistemática Tropical. Universidad de Sucre, Carrera 28 No. 5-267. Sincelejo, Colombia Autor de correspondencia: Colombia. jorge.mercado@unisucre.edu.co.

c Instituto Alexander von Humboldt. Villa de Leyva, Colombia. hmendoza@humboldt.org.co 


\section{Introducción}

El bosque seco tropical (bs-t) es uno de los ecosistemas más frágiles en todo el trópico [1]. Las condiciones climáticas y de fertilidad en sus suelos han facilitado el desarrollo de una variedad de actividades agrícolas, aspecto que lo ha fragmentado y degradado fuertemente [2]. En Colombia el bs-t, no sólo se encuentra amenazado por la agricultura, también por el constante avance de la ganadería y el urbanismo, a tal punto que sólo resta menos del 4\% de su cobertura original [3].

En Colombia este ecosistema se encuentra principalmente en la zona norte del país y hace parte del cinturón árido pericaribeño [4]. En este se encuentran los departamentos de Sucre y Bolívar, en los cuales se localiza uno de los fragmentos con mayor extensión de bs-t en el Caribe y en buen estado de conservación [3]. En esta área se han declarado varias reservas para la protección de estos bosques, entre ellas la Reserva Forestal Protectora Serranía de Coraza y Montes de María con una superficie de 6,653ha [5], localizadas en los municipios de Toluviejo, Colosó y Chalán en el departamento de Sucre. No obstante, en la actualidad hay un acelerado avance de los procesos antrópicos como los ya mencionados e incluyendo la minería, que ha causado una gran pérdida de la diversidad existente en los bosques de los Montes de María en el departamento de Sucre [6]. En esta área se han realizado algunos trabajos sobre la diversidad biótica como los de Galvan, et al. [7] y Patiño y Rangel [8], los cuales sólo se han enfocado en inventariar la vegetación de la Estación Primatológica en el municipio de Colosó, ubicada dentro de la reserva forestal, pero no existen estudios detallados que evalúen y comparen la composición y estructura de la vegetación fuera los límites de la reserva.

Teniendo en cuenta lo anterior, en el presente artículo se abordan los resultados sobre composición y estructura de un estudio mayor que analiza los patrones de diversidad, distribución y ecología en los bs-t de siete localidades en los Montes de María en el departamento de Sucre.

\section{Materiales y Métodos}

\section{1. Área de estudio}

Se localiza en la formación montañosa llamada Montes de María (tabla 1) ubicada entre los departamentos de Bolívar y Sucre en el Caribe colombiano (tabla y figura 1).

Tabla 1. Puntos de muestreo con sus respectivos municipios, nombres de localidad y altitud en los Montes de María (Sucre).

\begin{tabular}{|c|c|c|c|c|}
\hline Puntos & Municipio & Localidad & Coordenadas & $\begin{array}{c}\text { Altitud } \\
\text { (m.s.n.m) }\end{array}$ \\
\hline P1 & Morroa & $\begin{array}{c}\text { Casa Amarilla } \\
\text { uno }\end{array}$ & $\begin{array}{l}9^{\circ} 25^{\prime} 25.01^{\prime \prime} \mathrm{N} \\
75^{\circ} 19^{\prime} 4.07 " \mathrm{O}\end{array}$ & 202 \\
\hline P2 & Morroa & $\begin{array}{c}\text { Casa Amarilla } \\
\text { dos }\end{array}$ & $\begin{array}{r}9^{\circ} 26^{\prime} 11.79 " \mathrm{~N} ; \\
75^{\circ} 19^{\prime} 11.23^{\prime \prime} \mathrm{O} \\
9^{\circ} 30^{\prime} 4.33^{\prime \prime} \mathrm{N}\end{array}$ & 180 \\
\hline P3 & Colosó & Paraíso & $\begin{array}{c}75^{\circ} 23^{\prime} 11.57^{\prime \prime} \mathrm{O} \\
9^{\circ} 31^{\prime} 22.06^{\prime \prime} \mathrm{N} ;\end{array}$ & 472 \\
\hline P4 & Colosó & Pajarito & $\begin{array}{l}75^{\circ} 22^{\prime} 45.33^{\prime \prime} \mathrm{O} \\
9^{\circ} 34^{\prime} 46.32^{\prime \prime} \mathrm{N}\end{array}$ & 194 \\
\hline P5 & Chalán & Garrapata & $\begin{array}{l}75^{\circ} 20^{\prime} 3.06^{\prime \prime} \mathrm{O} \\
9^{\circ} 39^{\prime} 19.19^{\prime \prime} \mathrm{N}\end{array}$ & 611 \\
\hline P6 & San Onofre & El Cacao & $\begin{array}{r}75^{\circ} 23^{\prime} 50.71 " \mathrm{O} \\
9^{\circ} 31^{\prime} 6.911 \mathrm{~N}\end{array}$ & 380 \\
\hline P7 & Toluviejo & Roca Madre & $75^{\circ} 23^{\prime} 22.39^{\prime \prime} \mathrm{O}$ & 500 \\
\hline
\end{tabular}

Estos montes tienen una extensión de $6.297 \mathrm{Km}^{2}$, dentro de los cuales $2.578 \mathrm{Km}^{2}$ corresponden al departamento de Sucre, y alcanzan una elevación de hasta $700 \mathrm{~m}$ [5] (figura 1).

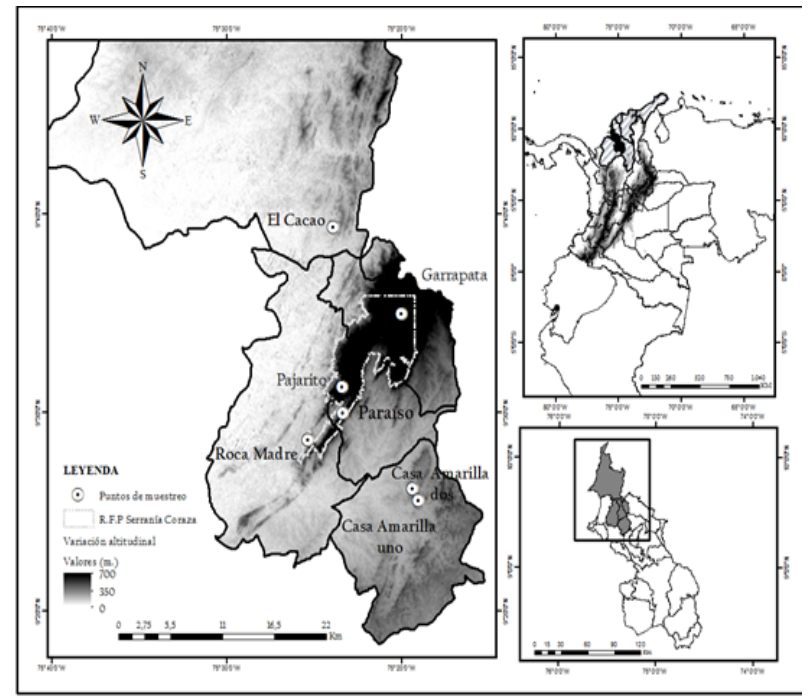

Figura 1. Disposición de los puntos de muestreo sobre el rango altitudinal (0-700 msnm) en los Montes de María del departamento de Sucre.

El clima del área está influenciado por los vientos Alisios de norte-este, producto de la proximidad al mar Caribe, y por el relieve montañoso y de colinas de la serranía de San Jacinto [9]. Estas condiciones han generado un clima cálido, en el cual la temperatura varía entre los $26^{\circ} \mathrm{C}$ y $30^{\circ} \mathrm{C}$ [10]. La precipitación anual es de $1,500 \mathrm{~mm}$ y la humedad relativa se encuentra entre $75 \%$ y $85 \%$. El régimen pluviométrico es bimodal, con valores máximos en los meses de septiembre a noviembre y mayo a junio [9]. 
El tipo de vegetación en el área corresponde a bosque seco tropical según Holdridge [11]. Algunos estudios desarrollados han mencionado que esta área hace parte del Zonobioma Tropical Alternohígrico [12], compuesto principalmente por bosques de ladera y galería, caracterizados por presentar adaptaciones a suelos calcáreos, condición que señala unidades vegetales endémicas en la zona $[4,13]$.

\subsection{Evaluación de la vegetación}

Se establecieron siete puntos en donde se realizaron muestreos para determinar la estructura y composición en los Montes de María. Par establecer los puntos se escogieron grandes áreas a lo largo de la zona de muestreo a través de un análisis fotointerpretativo de imágenes Landsat 8 ETM del servicio geológico de los Estados Unidos (USGS) con resolución espacial de $30 \mathrm{~m}$ (http://www.usgs. gov/) y se definieron unidades evaluativas empleando un muestreo al azar estratificado [14]. Luego se elaboraron "shape files" de cada unidad utilizando el programa ArcGis 10.3 [15] para la ubicación de fragmentos y selección de los puntos de muestreo (figura 1), considerando el orden público en la región, la accesibilidad, el estado de conservación y las coberturas vegetales.

Una vez seleccionadas las áreas y puntos, se realizaron visitas preliminares con el fin de corroborar el estado de intervención antrópica en la vegetación. En los puntos seleccionados se realizaron inventarios con el método RAP de Gentry [16] y considerando las modificaciones propuestas por Álvarez, et al. [17] y Mendoza [18]. Este método consiste en realizar diez parcelas en línea recta de $50 \times 2 \mathrm{~m}$, distanciadas $25 \mathrm{~m}$ y distribuidas perpendicularmente a la pendiente; en cada subparcela se censan todas las plantas con diámetro a la altura del pecho (DAP) $\geq$ $1 \mathrm{~cm}$ y altura mínima de $1,5 \mathrm{~m}$; de los individuos se registra el hábito de crecimiento, se estima la altura y se recolectan 3 muestras por individuos para su posterior herborización.

Una vez herborizado el material, éste fue determinado lo más exacto posible empleando claves taxonómicas [19-25] o por comparación con ejemplares depositados en los Herbario del Jardín Botánico de Medellín Joaquín Antonio Uribe (Herbario JAUM) y Federico Meden Bogotá (FMB) del Instituto Alexander von Humboldt. Una vez identificados los ejemplares, se corroboró su determinación comparando con imágenes tipos en Jstor Plant Science (http://www.jstor.org/), ejemplares de Tropicos (http://www.tropicos. org/), United States Botanical Garden (http://www. usbg.gov/search-collection), y Herbario Nacional Colombiano (http://www.biovirtual.unal.edu.co). Finalmente, en el caso de aquellos individuos en el que no fue posible alcanzar su afinidad taxonómica, estas fueron revisadas e identificadas por especialistas. Una vez identificados, los ejemplares se depositaron en el Herbario de la Universidad de Sucre (HEUS) bajo la enumeración de F. Herazo.

\subsection{Análisis de la información}

En cada punto de muestreo se identificaron los hábitos de crecimiento predominante y se calcularon parámetros como la densidad (no. Individuos en 0.1 ha), frecuencia (número de subparcelas en donde se encuentra una especie), dominancia (sumatoria de las áreas basales de una especie) y los respectivos valores relativos. Con esto valores se estableció el Índice de valor de importancia por familia (IVF) y especie (IVI) de acuerdo a Rangel y Velásquez [26]. En este sentido, se comparó entre localidades la composición de las primeras cinco familias y especies con mayor IVF e IVI.

Para comparar la estructura horizontal y vertical para todos los puntos muestreados, los individuos censados se agruparon en tres clases de altura y diámetro: alturas - I) individuos de 1 a $5 \mathrm{~m}$, II) individuos de 5,1 a $10 \mathrm{~m}$ y III) individuos igual o mayor a $10 \mathrm{~m}$; diámetros del tronco a la altura del pecho - I) individuos de 1 a $16 \mathrm{~cm}$, II) individuos de 16,1 a $22 \mathrm{~cm}$ y III) individuos de 22,1 a 46 centímetros.

Una vez establecidas las clases, se obtuvieron los valores totales por punto de muestreo y se compararon empleando ANOVAs para establecer diferencias significativas $(P<0.05)$ [26]. En los casos donde el análisis de la varianza mostró variaciones significativas con respecto a las alturas, diámetros del tronco y área basal, se procedió a realizar una prueba estadística Tukey's entre localidades de cada clase. Esto con el fin de establecer diferencias significativas entre los sitios por clases. Lo cual fue posible a través de totales de cada subparcelas en la clase(s) correspondiente, empleando el programa Minitab 17.1.0 [27]. 


\section{Resultados}

\subsection{Riqueza y composición florística}

Se registró un total de 363 especies dentro de 196 géneros y 65 familias en los siete puntos de muestreo (Anexo 1). La familia y género con mayor riqueza fueron Fabaceae con 51 especies y Machaerium con 6 (Anexo 1); mientras Cynophalla verrucosa fue la especie con mayor abundancia.

De manera sintetizada la composición florística en cada una de las siete localidades de muestreo (tabla 2).

Tabla 2. Densidad y riqueza florística de los siete puntos de muestreo en los Montes de María (Sucre).

\begin{tabular}{lcc}
\hline Localidad & N. Individuos & Riqueza específica \\
\hline Casa Amarilla uno & 429 & 79 \\
Casa Amarilla dos & 365 & 73 \\
Paraíso & 563 & 120 \\
Pajarito & 385 & 110 \\
Garrapata & 534 & 104 \\
El Cacao & 334 & 111 \\
Roca Madre & 433 & 122 \\
\hline
\end{tabular}

\subsection{Hábitos de crecimiento}

En general, el hábito más predominante en el bosque seco de los Montes de María fue el arbustivo, seguido por árbol, liana, palma y en menor proporción hierba (figura 2). Sin embargo, algunos sitios mostraron variaciones puntuales con respecto a ciertos hábitos reportados. Tal es el caso de Casa Amarilla uno y dos, quienes fueron las únicas localidades en registrar el hábito hierba y no reportar el de palma (figura 3). Con respecto al hábito lianescente, Pajarito $(10,9 \%)$ fue la localidad con mayor representatividad; mientras que el de palmas fue mayor en El Cacao (2,7 \%) (figura 3).

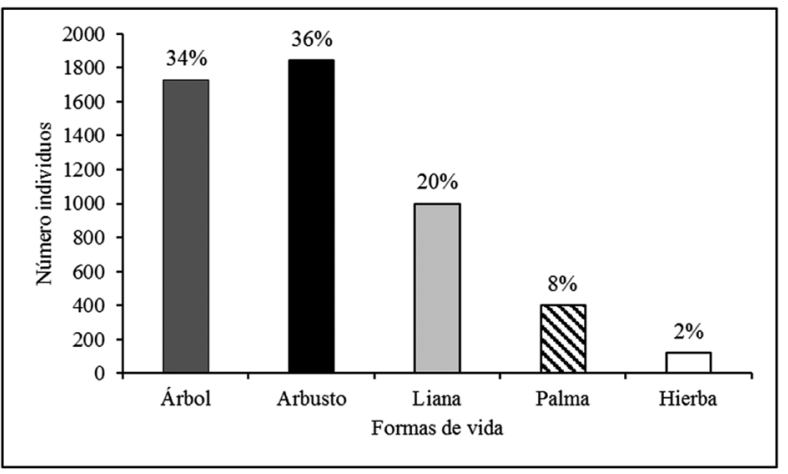

Figura 2. Comparación del número de individuos según las formas de vida presentes en el bosque seco de los Montes de María en el departamento de Sucre.

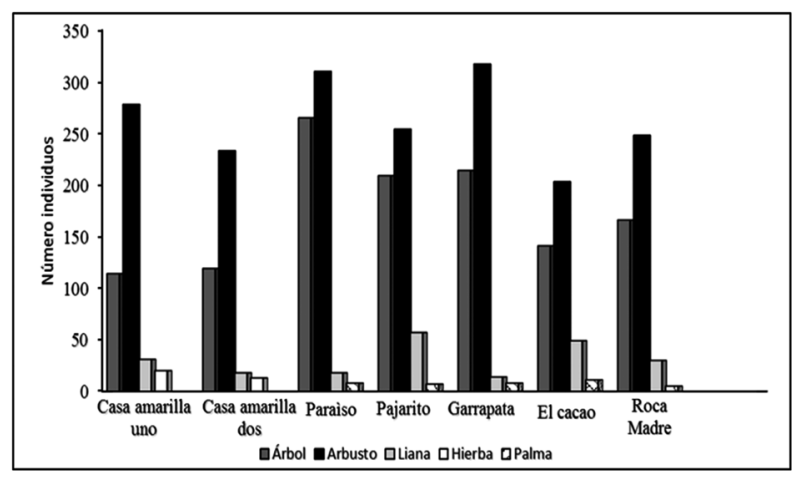

Figura 3. Comparación del número de individuos según las formas de vida presentes en cada localidad de los Montes de María en el departamento de Sucre.

\subsection{Estructura vertical y horizontal}

La altura máxima de los individuos encontrados en el muestreo fue de $37 \mathrm{~m}$. Al comparar las clases de alturas se encontraron diferencias significativas $(f$ $=9,103 ; p=0,0071)$ entre algunas localidades, en especial en la clase II. Las localidades de Paraíso, Pajarito y Garrapata presentaron el mayor número de árboles entre cinco y diez metros de altura con respecto a Casa Amarilla uno - dos y El Cacao (figura 4). Las mayores diferencias se observaron en el dosel del bosque con mayor número de individuos en las localidades de Paraíso, Pajarito y Garrapata, y menor número en Casa Amarillo dos.

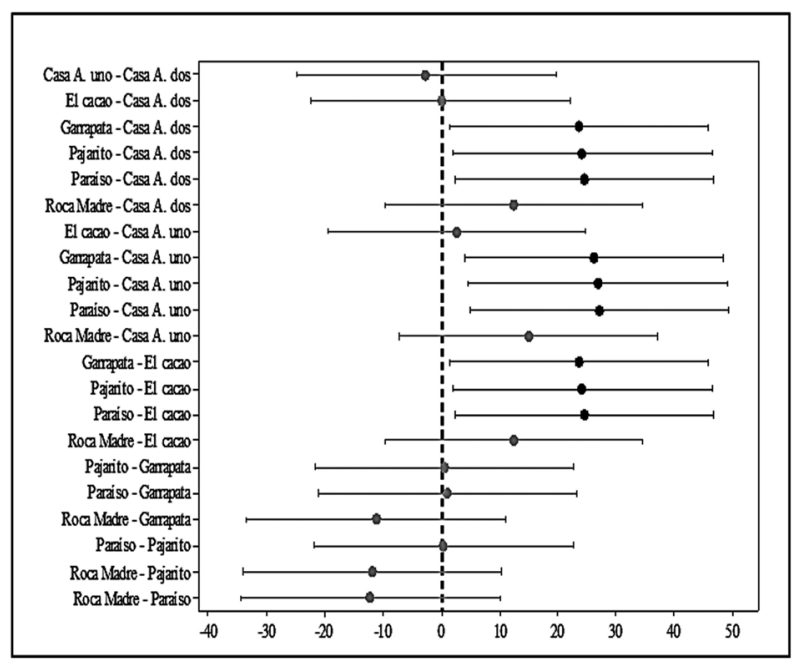

Figura 4. Clase altimétrica número II, diferencias de medias de las alturas entre localidades (según Tukey) en intervalos de confianza al $97 \%$. Intervalo de color negro que no contiene cero, las medias son significativamente diferentes.

Se encontró una tendencia en todas las localidades de mayor número de individuos en las dos primera clases de altura (I y II), que representan alturas entre 1-10 metros, con tendencia a disminuir en las de mayor altura (figura 5). La clase I reportó el $71 \%$ 
de los individuos de todos los muestreos, y en las localidades Garrapata, Paraíso y Casa Amarilla uno fue la categoría con mayor abundancia (figura 5).

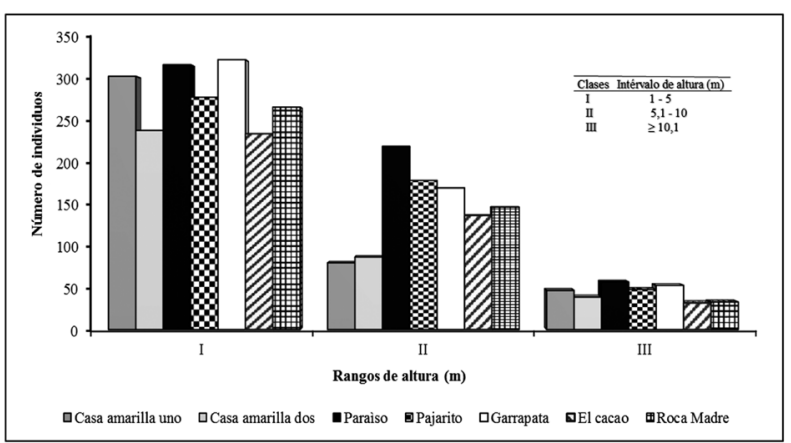

Figura 5. Relación del número de individuos según las clases altimétricas en los sitios de muestreo de los Montes de María en el departamento de Sucre.

La clase II de altura presentó el $19 \%$ de los individuos de todos los muestreos, con los valores más altos en las localidades de Paraíso, Pajarito y Garrapata (figura 5). Por ultimo en la clase III (árboles emergentes), se encontró un $10 \%$ de los todos los individuos, con mayor predominancia en la mismas localidades de la clase anterior (figura5).

Al igual que en los datos altimétricos, la distribución de los individuos por clases diamétricas mostró diferencias significativas entre localidades $(f=2,762 ; P=0,0064)$. En especial, en la clase III, Casa Amarilla uno presentó un mayor número de árboles de 22 a $46 \mathrm{~cm}$. de DAP con respecto a Casa amarillo dos, El Cacao y Roca Madre (figura 6). En esta misma clase, Paraíso mostró superioridad en relación a Casa Amarilla dos, El Cacao y Roca Madre (figura 6). Mientras que Garrapata también alcanzó valores significativos sobre Casa Amarilla dos y El Cacao (figura 6).

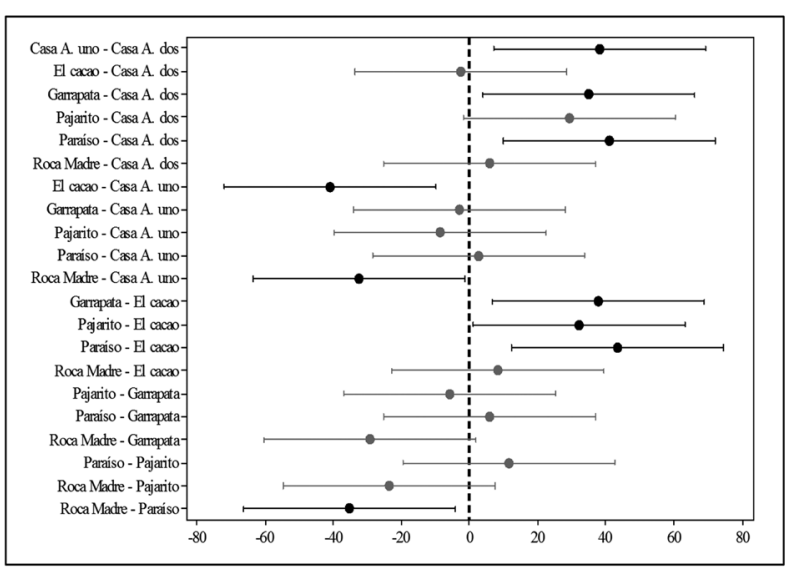

Figura 6. Clase diamétrica número III, diferencias de medias de DAP entre localidades (según Tukey) en intervalos de confianza al 97\%. Intervalos de color negro no contiene cero, las medias son significativamente diferentes.
Por otra parte, estas clases diamétricas también mostraron una tendencia semejante a las alturas. La clase diamétrica I fue la mayor número de individuos, seguida de la III y la de menor proporción la clase II (figura 7).

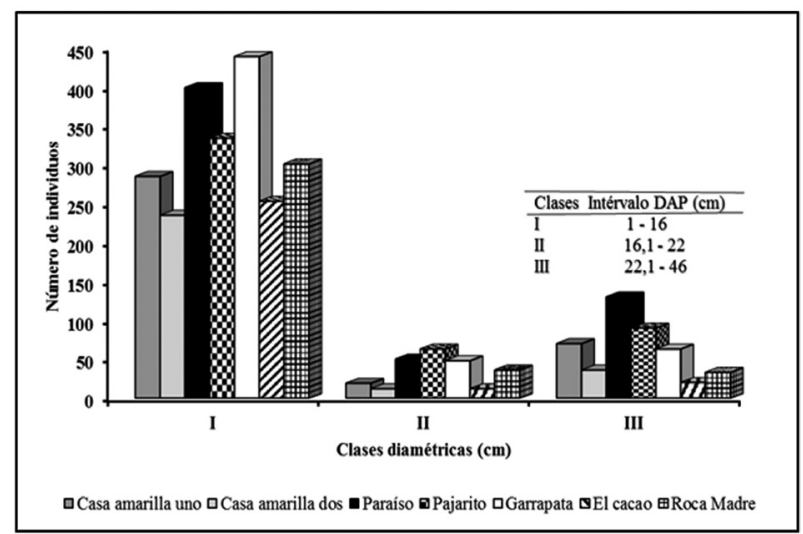

Figura 7. Relación del número de individuos según las clases diamétricas en los sitios de muestreo de los Montes de María en el departamento de Sucre.

El $75 \%$ de los individuos se acumuló en la clase diamétrica I, siendo Garrapata, Paraíso y Pajarito las localidades con la mayor abundancia en este rango (figura 7). En la clase II se registró el $10 \%$ de los árboles, con Paraíso, Pajarito y Garrapata, quienes reportaron los mayores porcentajes de individuos (figura 7). Por último, en la III, se acumuló el 15 $\%$ de los todos los individuos, y las localidades con mayor abundancia fueron Paraíso, Pajarito y Casa Amarilla uno (figura 7).

\section{4. Área basal}

El Área basal total de todos los muestreos fue de $19,58 \mathrm{~m}^{2} / 0.7 \mathrm{ha}$, con un rango de valores por localidad entre 1,7 y $3,8 \mathrm{~m}^{2} / 0.1$ ha y un promedio de $2,8 \mathrm{~m}^{2} / 0.1$ ha (tabla 3 ).

Tabla 3. Valores del Área basal en los muestreos realizados en los Montes de María del departamento de Sucre.

\begin{tabular}{lc}
\hline Localidad & $\begin{array}{c}\text { Total de área basal }\left(\mathrm{m}^{2} /\right. \\
0,01 \text { ha. }\end{array}$ \\
\hline Casa Amarilla uno & 2,80 \\
Casa Amarilla dos & 1,81 \\
Paraíso & 3,45 \\
Pajarito & 3,87 \\
Garrapata & 3,44 \\
El Cacao & 1,70 \\
Roca Madre & 2,51 \\
TOTAL & 19,58 \\
\hline
\end{tabular}


Las localidades de El Cacao, Roca Madre y Casa Amarilla dos presentaron los valores menores en el área basal, mientras que Casa Amarilla uno, Paraíso, Pajarito y Garrapata presentaron los mayores (figura 8). El Cacao fue la localidad con el valor más bajo de área basal y marcadas diferencias con respecto a las localidades con mayor cantidad de madera (figura 8).

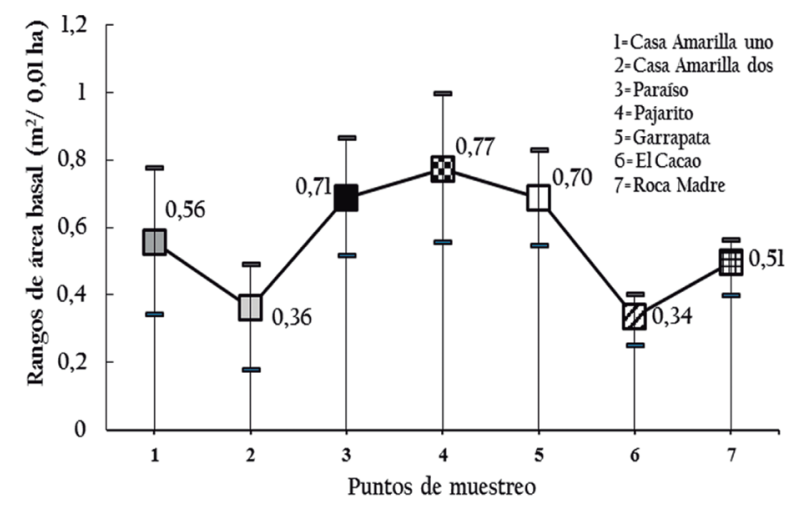

Figura 8. Promedios de área basal $\left(\mathrm{m}^{2}\right)$ en 0,01 ha de las siete localidades muestreadas en los Montes de María del departamento de Sucre.

Se encontraron diferencias estadísticamente significativas en una de las medias de las siete localidades $(F=5,048 ; P=0,008724)$, estas diferencias sólo se observaron al comparar las localidades en la clase diamétrica III. Casa Amarilla uno, Paraíso y Garrapata mostraron la mayor área basal (en la clase diamétrica III) con diferencia significativa con respecto a Casa Amarilla dos, El caco y Roca Madre (figura 9).

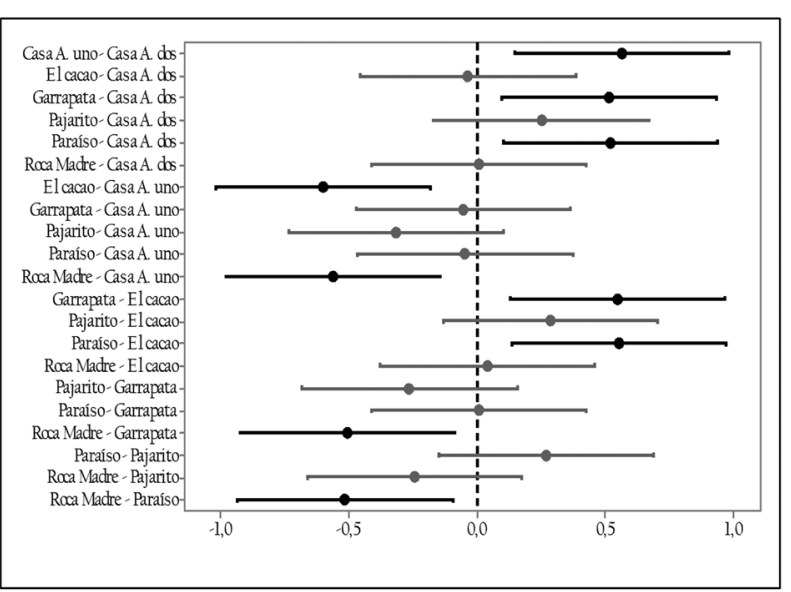

Figura 9. Comparación del Área basal (según Tukey) en intervalos de confianza al 97\%. Intervalo de color negro que no contiene cero, las medias son significativamente diferentes.

En la clase diamétrica III fue donde se encontró la mayor variación entre localidades. En esta Paraíso, Casa Amarilla uno, Pajarito y Garrapata mostraron la mayor cantidad de madera, mientras que en El
Cacao y Roca Madre la menor cantidad de madera, tal como se nota en la figura 10 .

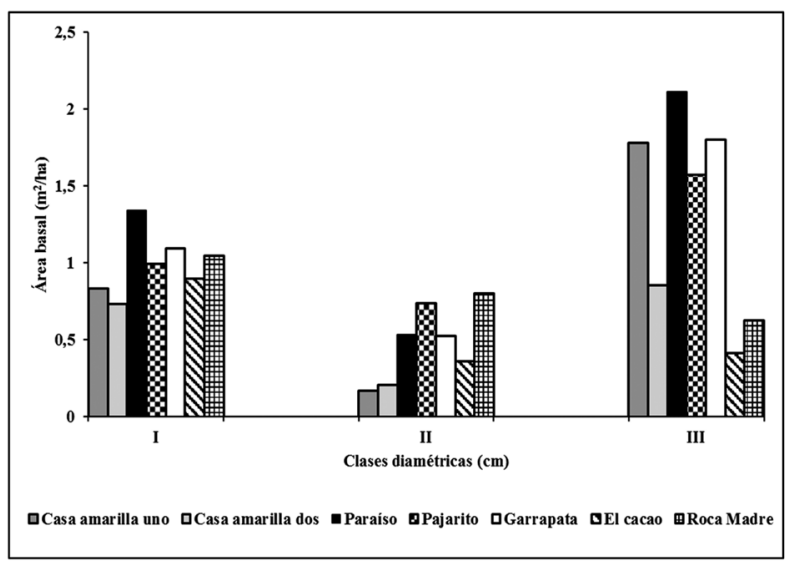

Figura 10. Relación del área basal en metros cuadrado según clases diamétricas $(\mathrm{cm})$ en los puntos de muestreo, Montes de María del departamento de Sucre.

\section{Valor de importancia de familias y especies}

Se analizaron las primeras cinco familias y especies con mayor índice de valor de Importancia por cada sitio de muestreo. La familia Capparaceae presentó los valores más altos de IVF, mientras que al nivel de especie Simira cordifolia presentó el mayor valor de importancia en todo el muestreo (tabla 4).

Las familias Fabaceae, Rubiaceae y en repetidos casos Capparaceae se registraron constantemente dentro de las primeras cinco con mayor importancia en todos los puntos de muestreo, mientras que Brosimum alicastrum y Simira cordifolia se observaron reiterativamente como especies importantes en todas las localidades (tabla 4).

\section{Discusión}

La variación registrada en la vegetación (363 especies, 196 géneros y 65 familias) de los Montes de María en el departamento de Sucre, es evidencia de la riqueza vegetal de los bosques secos del país. Esta composición fue similar a la encontrada en otros estudios $[18,28]$, lo cual permite afirmar que esta zona corresponde a bs-t.

Sin embargo, los resultados de riqueza pueden indicar que los Montes de María, puede ser una de las áreas de bosque seco con mayor diversidad, en comparación a otras localidades del país. Por ejemplo, en los departamentos de Atlántico y Bolívar, Rodríguez, et al. [28] reportaron un total de 314 especies; asimismo, Mendoza [18] para la región Caribe sólo estableció 236 especies y en el Magdalena 104, área en la cual Marulanda, et al. [29] registra años posteriores 155 taxones. 
Tabla 4. Primeras cinco familias y especies con mayor índices de valor de importancia (IVF - IVI) reportados en los Montes de María del departamento de Sucre.

\begin{tabular}{|c|c|c|c|}
\hline $\begin{array}{l}\text { Localidad/ } \\
\text { Familia }\end{array}$ & IVF & Especie & IVI \\
\hline \multicolumn{4}{|l|}{$\begin{array}{l}\text { Casa Amarilla } \\
\text { uno }\end{array}$} \\
\hline Capparaceae & 58,18 & Simira cordifolia & 31,59 \\
\hline Rubiaceae & 34,66 & Aspidosperma polyneuron & 22,80 \\
\hline Fabaceae & 29,10 & Melicoccus bijugatus & 20,29 \\
\hline Sapindaceae & 26,04 & Monilicarpa tenuisiliqua & 17,31 \\
\hline Apocynaceae & 26,02 & Quadrella odoratissima & 15,07 \\
\hline \multicolumn{4}{|c|}{$\begin{array}{l}\text { Casa Amarilla } \\
\text { dos }\end{array}$} \\
\hline Capparaceae & 52,01 & Simira cordifolia & 24,42 \\
\hline Rubiaceae & 40,02 & Trichilia acuminata & 24,36 \\
\hline Meliaceae & 21,96 & Cynophalla verrucosa & 19,78 \\
\hline Acanthaceae & 16,01 & Ampelocera edentula & 16,15 \\
\hline Fabaceae & 16 & Quadrella odoratissima & 12,28 \\
\hline \multicolumn{4}{|l|}{ Paraíso } \\
\hline Moraceae & 52,25 & Aspidosperma polyneuron & 23,38 \\
\hline Apocynaceae & 38,54 & Brosimum alicastrum & 23,03 \\
\hline Fabaceae & 31,42 & Sorocea sprucei & 17,07 \\
\hline Rubiaceae & 29,60 & Astronium graveolens & 12,09 \\
\hline Sapindaceae & 14,61 & Bursera simaruba & 8,839 \\
\hline \multicolumn{4}{|l|}{ Pajarito } \\
\hline Fabaceae & 41,56 & Brosimum alicastrum & 25,21 \\
\hline Moraceae & 26,66 & Bursera simaruba & 21,56 \\
\hline Bignoniaceae & 24,21 & Cynophalla verrucosa & 11,53 \\
\hline Sapindaceae & 16,54 & Pradosia colombiana & 8,466 \\
\hline Rubiaceae & 15,45 & Trichiliahirta & 8,320 \\
\hline \multicolumn{4}{|l|}{ Garrapata } \\
\hline Rubiaceae & 38,73 & Simira cordifolia & 23,58 \\
\hline Moraceae & 36,41 & Trichilia acuminata & 22,73 \\
\hline Meliaceae & 22,66 & Garcia nutans & 17,57 \\
\hline Apocynaceae & 22,34 & Brosimum alicastrum & 15,60 \\
\hline Fabaceae & 18,32 & Capparidastrum frondosum & 11,99 \\
\hline \multicolumn{4}{|l|}{ El Cacao } \\
\hline Salicaceae & 47,87 & Myrcia fallax & 23,07 \\
\hline Fabaceae & 20,30 & Machaerium capote & 15,80 \\
\hline Bignoniaceae & 18,05 & Centrolobium yavizanum & 14,94 \\
\hline Myrtaceae & 16,75 & Alseis labatioides & 9,174 \\
\hline Rubiaceae & 14,87 & Piperhispidum & 6,294 \\
\hline \multicolumn{4}{|l|}{ Roca Madre } \\
\hline Fabaceae & 38,85 & Ampelocera edentula & 15,31 \\
\hline Moraceae & 35,11 & Brosimum alicastrum & 11,66 \\
\hline Apocynaceae & 22,57 & $\begin{array}{l}\text { Tabernaemontana } \\
\text { amygdalifolia }\end{array}$ & 9,832 \\
\hline Rubiaceae & 21,24 & Astronium graveolens & 9,592 \\
\hline Euphorbiaceae & 19,51 & Stemmadenia grandiflora & 9,566 \\
\hline
\end{tabular}

La riqueza presente en los Montes de María puede deberse al producto de varios aspectos, el primero puede ser la orografía montañosa que permite la retención de humedad proveniente del mar Caribe [4, 30]. Este fenómeno proporciona la retención de hojas y por lo tanto mayores tiempos para su reproducción en comparación a otros ecosistemas secos, los cuales están sometidos a una sequía más frecuente $[9,30]$. Los suelos calcáreos es el segundo aspecto que podría explicar esta riqueza; Gentry [13] sugiere que en Colosó (Montes de María), los suelos calcáreos posiblemente influyen en una alta tasa de crecimiento en los árboles, lo cual refleja mayor complejidad del bosque. Este mismo autor argumentan que los bosques corresponde a una transición entre bosques húmedo a seco tropical, por lo que los niveles de diversidad son más altos que en los bosques secos de los valles interandinos y zonas más secas del Caribe [13]. No obstante, los datos de composición hallados en esta localidad y comparados con otras zonas de bosque seco, permiten sugerir que en términos generales a nivel de composición Montes de María corresponden a la formación de bosque seco tropical.

Finalmente, un tercer aspecto es la disponibilidad de agua, estos bosques están influenciados por una gran cantidad de arroyos y cuencas subterráneas que los mantiene gran parte del año hidratados, en otras palabras estas condiciones ambientales generan que exista menos estrés hídrico y por lo tanto una defoliación tardía e incluso carencia en algunas localidades como Colosó [5, 31-35].

Con respecto a la composición por familias, los bosques estudiados presentaron una composición típica de bosques secos en Colombia, en los cuales Fabaceae reporta el mayor número en especies (14, $26,28)$. Esta familia es altamente diversa en bosques húmedos y secos de zonas bajas $[2,36]$ y se atribuye a adaptaciones fisiológicas y estructurales, como la presencia de hojas compuestas, espinas o aguijones y varios tipos de hábitos de crecimiento [37].

Machaerium (Fabaceae) fue el género más diverso en todo el estudio. Este es un género de lianas y árboles de hojas compuestas, regularmente con aguijones asociados a las hojas $\mathrm{y}$ frutos samaroides fáciles de dispersar por el viento. Este predominio puede deberse a la adaptada morfología en los frutos y eficaz dispersión de semillas, aspecto que ha permitido establecerse desde la periferia de los bosques hasta el interior de los mismo, más aun permanecer en ecosistemas intervenidos [38]. La 
riqueza de este género coincide con lo reportado por Carrillo, et al. [39] en el Cerro Tasajero en el municipio de Cúcuta y por Mendoza [18] para el Valle del Magdalena y algunas localidades del Caribe.

Al nivel de especies, Cynophalla verrucosa (Capparaceae) fue la más abundante, dato reportado por primera vez en estos bosques, donde Capparaceae fue una de las familias con mayor valor ecológico y ha sido registrada como un grupo dominante y abundante en los Montes de María. La alta presencia de este grupo es de importancia, ya que esta puede ser utilizada según Cornejo y Iltis [40] como iniciador de procesos de restauración ecológica dada su resistencia a fuertes fluctuaciones hídricas en ecosistemas secos, es decir es un taxón siempre-verde que puede proveer sombra para grupos menos tolerantes en los biomas secos [13]. Esta familia, al igual que Rubiaceae han presentado una alta representatividad en los bosques del Caribe y en menos proporción en los valle interandinos del Magdalena [18, 29]

Con respecto a la riqueza por localidades, Roca Madre registró el mayor número de familias, géneros y especies (40, 92 y 122, respectivamente), posiblemente por pertenecer a la Reserva Forestal Protectora Serranía de Coraza y Montes de María (figura 1) [9] y a la presencias de arroyos que mantienen buenas condiciones de humedad durante gran parte del año. En esta zona también se reportó Brosimum alicastrum y Buxus citrifolia como especies más abundantes. La alta abundancia de $B$. alicastrum confirma lo propuesto por Pizano y García [3] ya que esta especie es señalada como indicadora de bosques poco intervenidos en fuertes pendientes, característica que coincide con los bosques en esta zona [41, 42]. En el caso de Buxus citrifolia, su alta abundancia la señalan, no sólo como un elemento importante dentro del bosque, también como, ya que esta especie solo crece en el área de estudio y el Darien (Panamá) focos de endemismo [4, 13]. En comparación con otras localidades, Roca Madre superó en especies a lo reportado en Tierra Bomba ( 56), Galerazamba (55) y Tayrona (67) [13, 18].

En cuanto al hábito de crecimiento, el arbustivo fue el más predominante en los Montes de María, esto puede deberse a que algunas especies arborescentes tienden a ser de copas reducidas, como respuesta al déficit de agua [3, 43], aspecto que facilita espacios que mantiene la buena iluminación en los estratos inferiores, favoreciendo el desarrollo en los individuos del sotobosque [44]. Sin embargo, los arboles también sobresalieron en algunas localidades (Casa Amarilla uno y Roca Madre), lo que puede indicar que estos bosques se encuentran en relativo buen estado de conservación [39, 45].

En el caso de Casa Amarilla uno y dos las clases diamétricas reflejan bosques de baja talla o posiblemente intervenidos; sin embargo al observar los datos de área basal $\left(2,80 \mathrm{~m}^{2} / 0,01\right.$ ha. $)$, estos reflejan una mayor similitud con los bosques secos de Tierra Bomba $\left(2.77 \mathrm{~m}^{2} / 0,01\right.$ ha. $)$ y con Forestal Monterrey (2.31 m²/ 0,01 ha.) [18], los cuales están caracterizados por presentar especies de porte bajo $[2,13,18]$. Del mismo modo, la carencia de suelos dominados por calizas y fuentes hídricas cercanas, pueden producir menor cantidad de nutrientes y estrés por déficit de agua, por lo tanto incrementa las tazas de defoliación y afecta el crecimiento arbóreo, en comparación con otras localidades como Paraíso, Pajarito y Roca Madre donde el bosque mostró mayor altura y área basal. Las causas anteriores generan que los sustratos inferiores (arbustivo y herbáceo), reciban mayor desarrollo [46]. Por su parte el habito lianescente demostró ser más representativo en Pajarito, lo que respalda la suposición de un lugar menos intervenido y con mejores condiciones como las ya mencionadas $[3,29]$.

Los datos altimétricos y diamétricos indicaron diferencias significativas entre localidades en las clase dos y tres respectivamente. Los sitios en mejor estado según estas clases fueron Paraíso, Pajarito y Garrapata, lo cual puede deberse a que estos se encuentran ubicados en un paisaje de montaña con suelos tipo II: arcillosos, poco drenados con calizas delEoceno-Mioceno y PH ligeramente ácidos a neutro $[4,47]$. Estos suelos pueden proveer los nutrientes necesarios para mejorar las tasas de crecimiento $\mathrm{y}$ desarrollo de estas comunidades. Mientras que las localidades más perturbadas (El Cacao con menos maderas y poca altura) se encuentran sobre un paisaje de lomerío, con suelos tipo IV: arcillas y areniscas muy drenados con PH ácidos, alterando en gran medida el funcionamiento y dinámica de estos bosques.

En cuanto al área basal, el valor total obtenido $\left(19,489 \mathrm{~m}^{2} / 0,1 \mathrm{ha}\right)$ se encontró entre los rangos (17 a $40 \mathrm{~m}^{2} / 0,1 \mathrm{ha}$ ) esperados para los bosques secos tropicales [2]. Este valor fue superior a lo encontrado en Neguaje $\left(11,40 \mathrm{~m}^{2} / 0,1 \mathrm{ha}\right)$ y Los Colorados 
(6.15 $\left.\mathrm{m}^{2} / 0,1 \mathrm{ha}\right)$. Según la distribución del área basal por clases diamétricas, los mayores valores se acumularon en la última clase (III) en los sitios de Casa Amarilla uno, Paraíso, Pajarito y Garrapata, aspecto que podría señalar a los Montes de María como buenos productores de biomasa [48]. Esto puede explicarse por el conflicto armado vivido en esta región, lo que ha garantizado la recuperación, permanencia y conservación vegetal del bosque durante los últimos 20 años [6].

Así mismo, se observaron resaltantes diferencias significativas en algunos promedios del área basal entre los puntos muestreados, como los registrados en Pajarito con relación a El caco. En Pajarito fueron notables mayores valores de área basal, con árboles de gran talla diamétrica (clase III), representados por Aspidosperma polyneuron, Brosimum alicastrum y Brosimum guianense, especies señaladas por presentar características maderables muy finas, y dentro de las cuales $B$. alicastrum obtuvo el mayor valor de importancia. Como fue mencionado anteriormente, los suelos calcaros observados en esta localidad pueden según Gentry [13] favorecer las tasas de crecimiento, con un aumento representativo, lo cual también explicaría la mayor área basal. Por el contrario, en El Cacao se mostraron los menores valores, con la presencia de Myrcia fallax y Machaerium capote, como taxones con alto valor de importancia. Estas especies han sido registradas en bosques con estado de sucesión temprana y de mucho follaje, pero de corto diámetro, que normalmente crecen en áreas sometidas a la minera $[49,50]$. Lo anterior es corroborado en las observaciones realizadas en campo, donde fue señalado que esta localidad (El Cacao) ha sido afectada en el pasado por el impacto humano y se encuentran en algún estado de sucesión [48], lo cual incluso al momento de realizar los muestreos generó inconvenientes ya que no fue posible establecer los transectos en fragmentos continuos.

\section{Conclusiones}

La composición es similar a la reportada en otros ecosistemas de bosque seco del país, sin embargo, la riqueza hallada en los Montes de María confirma lo propuesto por Pizano y García [3], quienes mencionan que estos bosques presentan un buen estado de conservación, teniendo en cuenta que actualmente existen fuertes impactos antrópicos en el área muestreada.
En términos de composición, Fabaceae fue la familia con mayor número de especies, aspecto que se mantiene para los bosques secos del Caribe $[18,28]$, el valle del Magdalena [18, 29] y el Cauca [51]. Así mismo se registraron otras familias de importancia ecológica, como Moraceae, Capparaceae y Rubiaceae, quienes mantienen también la tendencia de predominio en regiones secas del Neotrópico [13] y especialmente para Colombia [3, 18, 28, 29].

Con respecto a la riqueza por localidades, Paraíso (Colosó) se mostró como el segundo sitio con mayor numero especies, valor que superó (7 especies) lo reportado por Gentry [13] para el municipio de Colosó. Asimismo, fue en esta donde se mostraron los mayores porcentajes en área basal. Estos resultados son de gran importancia ya que permiten aclarar lo expuesto por Gentry [13] al mencionar que los bosques de los Montes de María podrían ser bosques en transición (seco a húmedo), en este sentido los resultados aquí descritos permiten determinar que estos son bosques secos tropicales, y que las variaciones existentes en su estructura, más que un reflejo de la humedad, puede deberse a otros elementos como los suelos.

En cuanto al DAP y cantidad de madera, los sitios Casa Amarilla uno Paraíso, Pajarito y Garrapata, pueden ser sugeridos como bosques menos perturbados en los Montes de María. Así mismo, es de importancia mencionar que las localidades de Casa Amarilla uno y dos deben permanecer bajo seguimiento constantes, ya que estas a pesar de estar tan cerca geográficamente pueden estar divergiendo, debido al comportamiento registrado en el DAP y área basal. Es de interés mencionar que éstas se encuentran bajo constante amenaza, debido a la alta transformación por acción de la ganadería y minería intensiva en la zona. También se recomienda intensificar el trabajo en las áreas ubicadas fuera de la reserva forestal (Norte del municipio de San Onofre), donde la acelerada transformación y la minería, incrementan la posibilidad de desaparición de estos bosques.

\section{Agradecimiento}

Se expresan agradecimientos a la División de investigaciones de la Universidad de Sucre por la financiación y prestamos de sus instalaciones. A la Fundación Luis Ángel Escobar por financiar parte de la investigación a través del programad de becas. A Pedro Álvarez, Hernando Gómez, Deiber Bertel y los 
estudiantes Tatiana Vergara, José Cobo Meza, Viviana Mejía Acosta, Samuel Angulo y Dairo Carrascal Prasca por el apoyo y esfuerzo en la implementación de los transectos y recolecta de la flora en campo; además del procesamiento del material en el Herbario HEUS. A Diego Zapata, Norberto López y Álvaro Cogollo en el Herbario del Jardín Botánico de Medellín, Joaquín Antonio Uribe (JAUM) por su colaboración en la corroboración e identificación de las especies.

\section{Referencias}

[1] P. Murphy y A. Lugo, Dry forests of Central America and the Caribbean, 1995, pp. 9-34.

[2] P. Murphy y A. Lugo, "Ecology of Tropical Dry Forest," Annual Review of Ecology and Systematics, vol. 17, pp. 67-88, 1986.

[3] C. Pizano y H. García, El Bosque Seco Tropical en Colombia. Instituto de Investigación de Recursos Biológicos Alexander von Humboldt (IAvH). Ediprint Ltda. ed. Bogota, 2014, p. 353.

[4] G. Halffter, "La diversidad biológica de Iberoamérica," Acta Zoológica Mexicana, vol. 1, p. 363, 1992.

[5] M. Aguilera, Montes de María: una subregión de economía campesina y empresarial, Documentos de trabajo sobre economía regional ed. Cartagena, 2013, p. 195.

[6] A. Sampedro, H. Gómez, y G. Ballut, "Estado de la vegetación en localidades abandonadas por "desplazamiento", en Los Montes De María Sucre, Colombia," Rev. Colombiana cienc. Anim., vol. 6, pp. 184-193, 2014.

[7] S. Galvan, I. Sierra, H. Gómez, J. De la ossa, y A. Patiño, "Biodiversidad en el área de influencia de la estación primates de Colosó, Sucre, Colombia.," Revista Colombiana de Ciencia Animal, vol. 1, pp. 95-118, 2009.

[8] R. Patiño y O. Rangel, "Estudio de caso. La vegetación boscosa en la estación de primates Coloso-Sucre. En O. Rangel-Ch (ed.). Colombia diversidad biótica XI. Patrones de la estructura y de la riqueza de la vegetación en Colombia. Bogotá: Universidad Nacional de Colombia (Sede Bogotá) Facultad de Ciencias.,"pp. 253-268, 2011.

[9] Promontes, Programa de Desarrollo y Paz de los Montes de María -Promontes, Corporación
Territorios, Universidad de Cartagena. Cartagena, 2003, p. 162.

[10] M. Aguilera, La economía del Departamento de Sucre: ganadería y sector público. Cartagena, 2005, p. 63.

[11] L. R. Holdridge, "Determination of World Plant Formations from Simple Climatic Data," Science, vol. 105, pp. 367-368, 1947.

[12] J.Hernández y H. Sánchez, "Biomas terrestre de Colombia. Pág.153-176. En: La biodiversidad biológica de Iberoamérica I. Halffter, G, Inst Mex. De Ecología y secretaría de Desarrollo Social. México DF.," 1992.

[13] A. Gentry, Diversity and floristic composition of neotropical dry forests, Cambridge University Press ed. vol. 85. Cambridge, 1995, pp. 146194.

[14] A. Newton, Forest Ecology and Conservation, Series Editor: William J. Sutherland ed. New York, 2007, p. 471.

[15] ESRI, “ArcGIS Desktop: Release 10.3 Redlands, CA: Environmental Systems Research Institute.," 2015.

[16] A. Gentry, "Patterns of neotropical plant species diversity," Evolution biology, vol. 15, pp. 1-84, 1982.

[17] E. Álvarez, A. Cogollo, H. Rincón, D. Benítez, T. Parra, W. Rodríguez, et al., "Propuesta metodológica de parcelas normatizadas para los inventarios de vegetación ISA-JAUM," Documento de trabajo.Versión 1.7. Medellín, Colombia, 2001.

[18] H. Mendoza, "Estructura y riqueza florística del bosque seco tropical en la región caribe y el valle del río magdalena, colombia," Caldasia, vol. 21, pp. 70-94, 1999.

[19] H. Mendoza, B. Ramírez, y L. Jiménez, Rubiaceae de Colombia, Ramos López Editorial ed. Bogota, 2004, p. 351.

[20] A. Gentry, Flora de Colombia: Bignoniaceae, Arfo Editores ed. Universidad Nacional de Colombia, Fac. de Ciencias, Instituto de Ciencias Naturales.Bogota, 2009, p. 462.

[21] E. Forero y C. Romero, Estudios en leguminosas colombianas, Academia colombiana de ciencias exactas, físicas y naturales ed. Bogota, 2005, pp. 1-174. 
[22] F. Morales, "Estudios en las Apocynaceae neotropicales XXXIX: revisión de las Apocynoideae y Rauvolfioideae de Honduras," Anales del Jardín Botánico de Madrid, vol. 66, p. 45, 2009.

[23] L. Smith y P. Fernandez, "Revisio violacearum colombiae," Caldasia, vol. 6, pp. 83-181, 1954.

[24] A. Gentry, A field guide to the families and genera of woody plants of northwest South America (Colombia, Ecuador, Perú) with supplementary notes on herbaceous taxa, University of Chicago Press edition ed. Washington, DC, 1993, p. 895.

[25] O. Rangel, Colombia Diversidad Biótica XII. La Región Caribe de Colombia,Universidad Nacional de Colombia-Instituto de Ciencias Naturales. xxii, Editorial CÓDICE Ltda. ed. Bogotá, 2012, p. 1046.

[26] O. Rangel y A. Velásquez, Métodos de estudio de la vegetación, Editorial Guadalupe Ltda ed. Bogotá, 1997, pp. 59-82.

[27] I. Minitab, "Minitab (Programa de Cómputo) Versión 17.1.0 Estados Unidos,” 2013.

[28] G. Rodríguez, K. Banda, S. Reyes, y A. Estupiñán, "Lista comentada de las plantas vasculares de bosques secos prioritarios para la conservación en los departamentos de Atlántico y Bolívar (Caribe colombiano)," Biota Colombiana, vol. 13, pp. 7-39, 2012.

[29] L. O. Marulanda, A. Uribe, P. Velásquez, M. A. Montoya, A. Idárraga, M. C. López, et al., "Estructura y composición de la vegetación de un fragmento de bosque seco en San Sabastián, Magdalena (Colombia). I. Composición de plantas vasculares," Actualidades Biologicas, vol. 25, pp. 17-30, 2003

[30] J. Díaz, Región Caribe de Colombia. Colección ecológica del Banco de Occidente vol. 978. Cali, 2014, p. 12.

[31] A. López, "Esquema de ordenamiento territorial del municipio de Morroa, documento técnico," Directivos de la alcaldía de Morroa, pp. 63-70, 2002.

[32] D. Narváez, "Plan de desarrollo "Toluviejo somos todos" alcaldía de Toluviejo," Directivos de la alcaldía de Toluviejo, p. 184, 2012.

[33] N. Pineda, "Plan de Desarrollo "Primero la Gente" Alcaldía de San Onofre," Directivos de la alcaldía de San Onofre, p. 68, 2012.
[34] H. Tejada, "Esquema de ordenamiento territorial del municipio de Colosó," Ministerio de Ambiente, Vivienda y Desarrollo Territorial pp. 28-38, 2009.

[35] C. Verbel, "Plan de desarrollodel municipio de Colosó 2008- 2011 “ Alcaldía de Colosó, p. 170, 2008.

[36] A. Gentry, "Changes in plant community diversity and floristic composition on environmental and geographical gradients," Annals Missourl Botanical Garde, vol. 75, pp. 1-34, 1988.

[37] A. Ceroni, "Distribución de las Leguminosas de la parte alta de la Cuenca La Gallega. Morropón. Piura.," Ecología Aplicada, vol. 2, pp. 9-13, 2003.

[38] Meléndez A y P. A, "Synopsis of the genus Machaerium Pers.(LeguminosaePapilionoideae-Dalbergieae) in Venezuela," Acta Botánica Venezuelica, pp. 363-416, 2009.

[39] M. Carrillo, O. Rivera, y R. Sánchez, "Caracterización florística y estructural del bosque seco tropical del cerro Tasajero, San José de Cúcuta-norte de Santander, Colombia," Actualidades Biológica, vol. 29, pp. 55-73, 2007.

[40] X. Cornejo y H. Iltis, Flora de Jalisco y áreas colindantes; Capparaceae, Editores orgánica ed. Méjico, Universidad de Guadalajara, 2012, p. 71.

[41] S. Galván y J. De La Ossa, "Herpetofauna registrada para el área de influencia de la reserva forestal protectora serranía de Coraza, Coloso Sucre, Colombia," Revista Colombiana de Ciencia Animal, vol. 1, pp. 250-258pp, 2009.

[42] M. Lee y J. Rangel, “Composición florística del bosque tropical seco del santuario" Los Besotes" y fenología de especies arbóreas dominantes (Valledupar, Cesar, Colombia)," Colombia Forestal, vol. 18, pp. 87-103, 2015.

[43] E. Carbonó y H. García, "La vegetación terrestre en la ensenada de Neguanje, parque nacional natural Tayrona (Magdalena, Colombia)," Caldasia, vol. 32, pp. 235-256, 2010.

[44] R. Pennington, D. Prado, y C. Pendry, "Neotropical seasonally dry forests and Quaternary vegetation changes," Journal of Biogeography, vol. 27, pp. 261-273, 2000. 
[45] M. K. Guariguata, G. Kandler, M. Guariguata, y R. Manuel, Ecología y conservación de bosques neotropicales, Editorial Tecnológica ed. Costa Rica, 2002, p. 691.

[46] D. Olascuaga, J. Mercado, y L. Sánchez, "Análisis de la vegetación sucesional en un fragmento de bosque seco tropical en ToluviejoSucre (Colombia)," Colombia forestal, vol. 19, pp. 23 - 40, 2015.

[47] IGAC, Estudio general de suelos y zonificación de tierras del departamento de Sucre, Instituto Geográfico Agustín Codazzi. ed. Bogotá, 1998, p. 126.

[48] B. Louman, "Bases ecológicas. en: Silvicultura de bosques latifoliados húmedos con énfasis en América Central. Editado por: Louman, B; Quirós, D; Nilsson, M. Turrialba, CR,," Catie, pp. $57-622001$.
[49] David H, Díaz V, Urrea L, y C. N, Guía Ilustrada Flora Cañón del río Porce, Antioquia. EPM E.S.P. Universidad de Antioquia, Herbario Universidad de Antioquia. Medellín, Colombia, pp. 2642014.

[50] A. Vargas, Á. González, E. Barona, y W. Bolívar, "Composición y estructura vegetal de fragmentos de bosque seco tropical y de dos zonas con actividad antrópica en La Dorada y Victoria, Caldas," Revista de Ciencias, vol. 20, pp. 13-60, 2016.

[51] A. Torres, J. Bautista, M. Cárdenas, J. Vargas, V. Londoño, K. Rivera, et al., "Dinámica sucesional de un fragmento de bosque seco tropical del Valle del Cauca, Colombia," Biota Colombiana, vol. 13, pp. 66-84, 2012. 